\title{
Acúmulo de serapilheira e nutrientes em área com diferentes metodologias de Restauração Florestal
}

\author{
Valéria Hollunder Klippel', José Eduardo Macedo Pezzopane², Marcos Vinicius Winckler Caldeira²*, \\ Gilson Fernandes da Silva², Kallil Chaves Castro²
}

'Universidade Federal de Viçosa, Viçosa, MG, Brasil

${ }^{2}$ Universidade Federal do Espírito Santo, Alegre, ES, Brasil

*Autor correspondente, e-mail: mvwcaldeira@gmail.com

\begin{abstract}
Resumo
O objetivo desse estudo foi analisar os indicadores de restauração florestal (atributos químicos do solo, serapilheira acumulada e o conteúdo de nutrientes) em diferentes métodos de restauração florestal de uma Floresta Atlântica de Tabuleiro - Linhares, ES. O experimento foi instalado agosto de 2007 em blocos casualizados, com quatro tratamentos e três repetições: T1 (Controle): Somente o controle de formigas cortadeiras; T2: Roçada manual seletiva, capina química seletiva, controle de formigas e as espécies tolerantes ao herbicida; T3: Idêntico ao tratamento 2, mas com o plantio de espécies pioneiras da Mata Atlântica no espaçamento de $5 \times 5 \mathrm{~m}$, intercalado, no mesmo espaçamento, o semeio de Sesbania grandiflora em covas; e T4: Idêntico ao tratamento 2, mas com o plantio de 54 espécies da Mata Atlântica no espaçamento próximo de $3 \times 3 \mathrm{~m}$. A produção média total de serapilheira acumulada nos diferentes métodos de restauração florestal foi de $7,4 \mathrm{Mg} \mathrm{ha}^{-1}$. O conteúdo de $\mathrm{K}$ foi o único que apresentou diferença estatística (superior para o $\mathrm{Tl}$ é menor para o T4). Para os micronutrientes não foi encontrada diferença estatística na serapilheira acumulada entre os tratamentos avaliados. A avaliação de atributos químicos do solo e serapilheira acumulada de maneira geral, não foram influenciados pelas metodologias de restauração florestal, o que pode ser atribuído ao pouco tempo de implantação dos tratamentos
\end{abstract}

Palavras-chave: Ciclagem de nutrientes; espécies pioneiras, práticas silviculturais.

\section{Litter and nutrient accumulation in areas with different methodologies of forest restoration}

\begin{abstract}
The objective of this study was to evaluate the forest restoration indexes (chemical soil attributes, accumulated litter and nutrients content) in different methods of forest restoration of an Atlantic Forest Tableland in Linhares, ES, Brazil. The experiment was installed on August, 2007 in a randomized block design with four treatments and three repetitions: $\mathrm{Tl}$ (Control)- only the control of leaf-cutting ants, T2: manual selective mowing, selective chemical weed control, control of ants and herbicidetolerant species; T3: same for treatment 2, but planting pioneer species from the Atlantic Forest spaced $5 \times 5 \mathrm{~m}$, interleaving, in the same spacing, the seeding of Sesbania grandiflora; T4: same for treatment 2, but planting 54 species from the Atlantic Forest spaced $3 \times 3 \mathrm{~m}$. The average total production of litter, accumulated from the four different methodologies for forest restoration was 7.4 $\mathrm{Mg} \mathrm{ha}^{-1}$. The $\mathrm{K}$ content was the only with statistic significant difference (higher for $\mathrm{Tl}$ and lower for T4). For micronutrients, no statistical difference between treatments was observed when the accumulatedlitter was evacuated. The evaluation of soil chemical properties and accumulated litter, in general, were not influenced by the used methodologies for forest restoration, which can be attributed to the short time of implementation of the treatments.
\end{abstract}

Keywords: Nutrient cycling; pioneer species, silvicultural practices 


\section{Introdução}

A restauração florestal deve propiciar o restabelecimento das relações ecológicas e da sucessão vegetal em ambientes degradados. Em locais com limitações ambientais à sucessão de espécies, a regeneração natural pode ser potencializada por meio do plantio de espécies facilitadoras, sendo algumas das características desejáveis para essas espécies a capacidade de estabelecimento em condições limitantes, a atração da fauna, o crescimento rápido e a grande deposição de serapilheira, e capacidade de melhorar as condições do solo (Rodrigues et al., 2002; Chada et al., 2004).

A serapilheira é um componente de suma importância dentro de um ecossistema florestal, respondendo pela ciclagem de nutrientes, além de indicar a capacidade produtiva da floresta ao relacionar os nutrientes disponíveis com as necessidades nutricionais de uma dada espécie (Figueiredo Filho et al., 2003; Caldeira et al., 2008).

Por meio da ciclagem de nutrientes, via deposição de serapilheira, há um aumento do teor de matéria orgânica e da disponibilidade de nutrientes, a melhoria das propriedades físicas e a otimização da atividade biológica, que influenciam na qualidade dos solos (Franco \& Faria, 1997; Gama-Rodrigues et al., 1999; Caldeira et al., 2008).

A escolha inicial de espécies florestais para a formação de maciços pode refletir-se na sazonalidade, na quantidade e na qualidade de serapilheira produzida, interferindo nas características químicas do solo e na cadeia alimentar resultante dos detritos por elas gerados (Poggianl \& Schumacher, 2000; Caldeira et al., 2008).

A serapilheira é influenciada pelo tipo de vegetação, do estádio sucessional, da posição geográfica, da variação climática, das características químicas do solo e da atividade biológica, a mesma pode ser considerada um bioindicador de reação, por ter seus padrões modificações por alterações no meio (Machado et al., 2004).

Nesse contexto, esse estudo teve como objetivo quantificar o estoque de serapilheira acumulada e o conteúdo de nutrientes em diferentes métodos de restauração florestal em área de influência da Floresta Atlântica de Tabuleiro - Linhares, ES.

\section{Material e Métodos}

O presente estudo foi realizado na Reserva Natural Vale (RNV), $30 \mathrm{~km}$ ao norte do município de Linhares - Espírito Santo entre as $19^{\circ}$

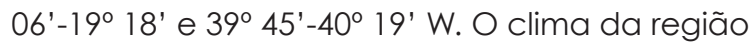
é do tipo Awi, tropical quente e úmido, com estação chuvosa no verão e seca no inverno. A precipitação pluviométrica média anual é de $1.202 \mathrm{~mm}$ e a temperatura média anual é de $23,3^{\circ} \mathrm{C}$, com média mínima de $14,8^{\circ} \mathrm{C}$ e máxima de $34,2^{\circ} \mathrm{C}$. A umidade relativa apresenta pouca variabilidade com médias anuais de 80,6 a $86,6 \%$ (Jesus \& Rolim, 2005). A Floresta de Tabuleiro na RNV é classificada como Floresta Ombrófila Densa de Terras Baixas (IBGE, 2012). O solo da RNV é classificado como Argissolo Amarelo (EMBRAPA, 2006).

A área de estudo, anteriormente ocupada com um plantio de eucalipto, apresentava-se coberta com gramíneas (principalmente sapê - Imperata brasiliensis Trin.) e algumas árvores isoladas em regeneração, onde as principais espécies encontradas foram Albizia polycephala (Benth.) Killip, Acacia auriculiformes, Acacia mangium, Aegiphila integrifolia (Jacq.) Moldenke, Astronium concinnum (Engl.) Schott, Astronium graveolens Jacq., Cecropia pachystachya Trécul., Couratari asterotricha Prance, Piptadenia paniculata Benth., Solanum sooretamum Carvalho e Sparattosperma leucanthum (Vell.) K. Schum.

$\mathrm{O}$ experimento foi instalado em agosto de 2007, em blocos casualizados com quatro tratamentos e três repetições, ocupando uma área total de 7,02 ha, onde cada repetição tem $65 \mathrm{~m} \times 90 \mathrm{~m}$. No entanto, a área útil de cada parcela é $50 \mathrm{~m} \times 40 \mathrm{~m}$, existindo uma borda entre um tratamento e outro.

Os seguintes tratamentos foram aplicados no momento da implantação: Tl (Controle): Somente o controle de formigas cortadeiras; T2: Roçada manual seletiva, capina química seletiva, controle de formigas e as espécies tolerantes ao herbicida, como algumas lianas ocorrentes, foram destocadas; T3: Idêntico 
ao tratamento 2, mas com o plantio aleatório de mudas de espécies pioneiras da Mata Atlântica no espaçamento de $5 \mathrm{~m} \times 5 \mathrm{~m}$, intercalando, no mesmo espaçamento, o semeio de Sesbania grandiflora em covas (10 sementes por cova). Portanto, o espaçamento final para este tratamento foi de $2,5 \times 2,5 \mathrm{~m}$; e T4: Idêntico ao tratamento 2, mas com o plantio aleatório de mudas de 54 espécies da Mata Atlântica no espaçamento de $3 \mathrm{~m} \times 3 \mathrm{~m}$.

Nos tratamentos 3 e 4 , onde houve plantio de mudas, procedeu-se a fertilização mineral composta por $200 \mathrm{~g}$ de superfosfato simples $18 \%$ de $\mathrm{P}_{2} \mathrm{O}_{5}, 16 \%$ de Cálcio e $8 \%$ de Enxofre), aplicado na cova no momento do plantio. Para manutenção dos tratamentos após a sua implantação, as seguintes atividades foram realizadas (Tabela 1):

Tabela 1. Atividades realizadas para manutenção dos tratamentos durante o primeiro, segundo e terceiro anos após a implantação do experimento na Reserva Natural Vale, Linhares - ES.

\begin{tabular}{|c|c|c|c|c|}
\hline \multirow{2}{*}{ Tratamento } & \multirow{2}{*}{ Atividade } & $1^{\circ}$ ano & $2^{\circ}$ ano & $3^{\circ}$ ano \\
\hline & & \multicolumn{3}{|c|}{$N^{\circ}$ de vezes } \\
\hline 1 & Controle de formiga & 1 & 1 & 1 \\
\hline \multirow{3}{*}{2} & Controle de formiga & 2 & 2 & 2 \\
\hline & Roçada manual seletiva & 4 & 3 & 2 \\
\hline & Aplicação seletiva de herbicida & 2 & 1 & 1 \\
\hline \multirow{4}{*}{3} & Controle de formiga & 2 & 2 & 2 \\
\hline & Coroamento de mundas e no semeio & 2 & 2 & 1 \\
\hline & Roçada manual seletiva & 4 & 3 & 2 \\
\hline & Aplicação seletiva de herbicida & 2 & 1 & 1 \\
\hline \multirow{4}{*}{4} & Controle de formiga & 2 & 2 & 2 \\
\hline & Coroamento de mundas & 2 & 1 & 1 \\
\hline & Roçada manual seletiva & 4 & 3 & 2 \\
\hline & Aplicação seletiva de herbicida & 2 & 1 & 1 \\
\hline
\end{tabular}

Para analisar os atributos químicos do solo nos diferentes tratamentos, foram coletadas amostras de solo nas profundidades 0 - 5; 5 - 10; $10-20$ e $20-40 \mathrm{~cm}$ em novembro de 2010. Foram coletadas de forma sistemática cinco subamostras em cada parcela e em cada profundidade. A sistematização foi definida coletando subamostras nos quatro cantos e no centro da área útil de cada parcela. Com auxílio de um enxadão foram abertas covas de aproximadamente $40 \mathrm{~cm}$ de profundidade e, utilizando uma pá de jardineiro, as subamostras foram retiradas em cada profundidade da cova e depositadas em baldes previamente identificados. Uma régua foi inserida verticalmente dentro da cova auxiliando no momento da coleta do solo para que os intervalos das profundidades $10-5 ; 5$ - 10; $10-20$ e $20-40 \mathrm{~cm}$ ) fossem respeitados. Depois de retiradas as cinco subamostras nas quatro profundidades na parcela, essas foram depositadas por profundidade nos baldes e homogeneizadas. De cada balde foi retirada uma amostra composta por parcela em cada profundidade.
Para a análise de serapilheira foram coletas de forma sistemática 10 amostras de serapilheira acumulada em cada parcela, utilizando gabaritos de madeira de $25 \mathrm{~cm} \times 25$ $\mathrm{cm}$. As coletas foram realizadas em novembro de 2010.

Para determinação da biomassa das amostras de serapilheira acumulada, as 10 amostras de cada parcela foram colocadas separadamente em sacos de papel, secas em estufa de circulação e renovação de ar a $65^{\circ} \mathrm{C}$ até alcançar peso constante, e que foi aferida em balança de precisão $(0,01 \mathrm{~g})$. A quantidade de serapilheira acumulada cada parcela foi estimada para megagrama por hectare (Mg ha1).

Para realização da análise química da serapilheira acumulada, as 10 amostras de cada parcela após secas e pesadas, foram homogeneizadas e trituradas em moinho do tipo Wiley, passadas em peneiras de malha $1,0 \mathrm{~mm}$ (20 mesh) e armazenadas em frascos de vidros para subsequente análise dos teores de macro $(\mathrm{N}, \mathrm{P}, \mathrm{K}, \mathrm{Ca}, \mathrm{Mg}, \mathrm{S})$ e micronutrientes ( $\mathrm{Fe}, \mathrm{Cu}$, $M n, Z n, B)$ e carbono orgânico $\left(C_{\text {org }}\right)$ conforme 
metodologia descrita por Tedesco et al., (1995) e Miyazawa et al. (1999).

O conteúdo dos nutrientes e carbono orgânico contidos na serapilheira transferidos para o solo via deposição foi estimado utilizando a equação proposta por Cuevas \& Medina (1986).

Os dados obtidos de biomassa da serapilheira acumulada, teor e conteúdo dos nutrientes e carbono orgânico foram submetidos à análise de variância, e quando significativas, as médias foram comparadas pelo teste de Tukey, a $5 \%$ de probabilidade, sendo o software Matlab $6.5 \AA$ utilizado para as análises.

\section{Resultados e Discussão}

Atributos químicos do solo

Entre os tratamentos analisados para as profundidades de $0-5 \mathrm{~cm}$ e $20-40 \mathrm{~cm}$ não foram encontradas diferenças significativas para os atributos do solo analisados (Tabela 2).

Tabela 2. Resultado da análise química do solo na área de estudo - Reserva Natural Vale (RNV), Linhares - ES.

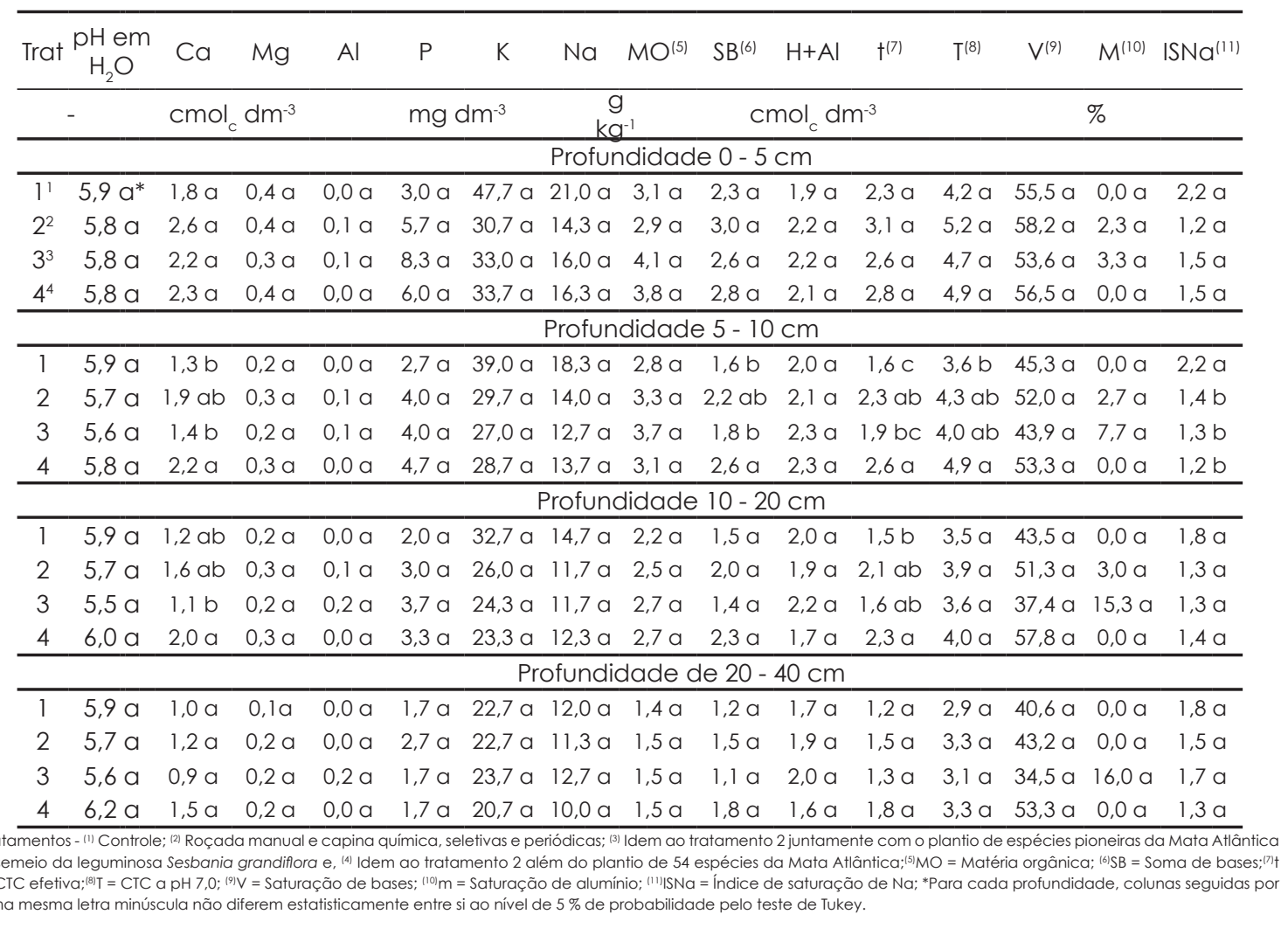

O teor de Ca foi significativamente maior no tratamento T4 em duas profundidades, em relação aos tratamentos $\mathrm{T} 1$ e T2 na camada 05-10 cm, e em relação ao tratamento $T 1$ na camada $10-20 \mathrm{~cm}$. O tratamento T4 também apresentou valor da CTC efetiva superior ao tratamento $\mathrm{Tl}$.

O melhor desempenho do T4 em relação aos demais tratamentos, pode estar associado a dois fatores: a adubação realizada antes do plantio e a maior diversidade.

A adubação, influenciou na diferença existente entre o T4 e o T1, onde apenas o T4 recebeu adubação durante o preparo da área, enquanto que o T1 não recebeu nenhum tipo de preparo, mantendo assim as condições semelhantes à implantação

De maneira geral, a área apresentou níveis baixos para os outros nutrientes, e para todos os tratamentos os valores dos atributos analisados tendem a decrescer em profundidade, como também foi observado por Moreau (2014) em área de Floresta de Tabuleiro no município de Aracruz, Espírito Santo.

No estudo realizado por Magnago et al. (2011) na Área de Proteção Ambiental (APA) da Lagoa de Jucunem, no município de Serra no Espírito Santo também em Floresta de 
Tabuleiro, os autores puderam observar que independente do estágio sucessional analisado, os solos eram semelhantes entre si, e mantinham a característica descrita.

Outros estudos realizados em solos de tabuleiros em áreas de recuperação florestal demonstraram que os solos dessas áreas, em geral, são de baixa fertilidade, baixa saturação por bases, e aumento da acidez em profundidade (Caló, 2014; Moreira, 2014).

\section{Serapilheira acumulada}

A biomassa da serapilheira acumulada não diferiv estatisticamente entre os tratamentos estudados. As médias para os tratamentos T1, T2, T3 e T4 foram, respectivamente, 5,7; 7,9; 9, 1 e 7,1 $\mathrm{Mg} \mathrm{ha}^{-1}$.

Os valores encontrados no presente estudo são similares a estudos realizados em Florestas Estacionais Semideciduais, variando entre 6,2 e 8,4 $\mathrm{Mg} \mathrm{ha}^{-1}$ (Vital, 2004). Porém, ressaltase que esses valores estão acima daqueles observados em outras tipologias florestais e em áreas que então em estágio inicial de formação. Caldeira et al. (2008) verificou um acúmulo de 4,5; 5,0 e 5,3 $\mathrm{Mg} \mathrm{ha}^{-1}$ em Floresta Ombrófila Densa Submontana, em Blumenau, Santa Catarina, respectivamente nos estágios inicial, intermediário e avançado de recuperação.

Segundo Fernandes et al. (2007), florestas em fase inicial de sucessão, pela grande presença de espécies pioneiras, depositam mais serapilheira, sendo o seu acúmulo o maior estoque de biomassa dessas formações, por outro lado, florestas em estado avançado de sucessão ou primárias conservam o maior estoque de biomassa na massa vegetal viva.

Esta afirmativa pode sugerir que apesar de não significativo, o maior acúmulo de serapilheira apresentado pelo T3, está relacionado à maior presença de espécies pioneiras neste tratamento em relação aos demais.

O acúmulo de serapilheira é influenciado por fatores ambientais, como temperatura e umidade; pela qualidade inicial do material formador como, por exemplo, pelos componentes orgânicos; pelos macro e micronutrientes; pelos organismos do solo, como fauna, actomicetos e bactérias (O'Connell \& Sankaran, 1997; Wedderburn \& Carter, 1999), além de variar em função da procedência, da espécie, da cobertura florestal, do estágio sucessional, da idade, da época da coleta, do tipo de floresta e do local. Além desses fatores, outros como: condições edafoclimáticas e regime hídrico, sítio, sub-bosque, manejo silvicultural, proporção de copa, bem como taxa de decomposição e distúrbios naturais como fogo e ataque de insetos ou artificiais como remoção da serapilheira e cultivos, ocorridos na floresta ou no povoamento, também influenciam no acúmulo de serapilheira (Caldeira et al., 2008).

Teores de macro e micronutrientes e carbono orgânico

Os teores médios de macronutrientes na serapilheira acumulada podem ser observados na Tabela 3, apresentando para o tratamento T1 a ordem decrescente: $\mathrm{Ca}>\mathrm{N}>\mathrm{Mg}>\mathrm{K}>\mathrm{S}$ $>\mathrm{P}$ e para os tratamentos T2, T3 e T4 a ordem decrescente: $\mathrm{Ca}>\mathrm{N}>\mathrm{Mg}>\mathrm{S}>\mathrm{K}>\mathrm{P}$. Não houve diferença estatística para os teores dos macronutrientes e $\mathrm{C}_{\text {org }}$ entre os tratamentos.

Tabela 3. Teores médios dos macronutrientes, carbono orgânico e relação $\mathrm{C} / \mathrm{N}$ da serapilheira acumulada nos diferentes métodos de restauração florestal de Mata Atlântica de tabuleiros, Linhares - ES

\begin{tabular}{|c|c|c|c|c|c|c|c|c|}
\hline \multirow{2}{*}{ Tratamento } & $\mathrm{N}$ & $P$ & $K$ & $\mathrm{Ca}$ & $\mathrm{Mg}$ & $S$ & $\mathrm{C}_{\text {ora }}$ & $\mathrm{C} / \mathrm{N}$ \\
\hline & \multicolumn{7}{|c|}{ Teor $\left(\mathrm{g} \mathrm{kg}^{-1}\right)$} & \\
\hline \multirow{2}{*}{1} & $10,51 a^{*}$ & $0,29 a$ & $1,17 a$ & $12,18 b$ & $1,91 a$ & $0,94 a$ & $422,33 a$ & $40,52 a$ \\
\hline & $( \pm 0,61)^{(1)}$ & $( \pm 0,01)$ & $( \pm 0,23)$ & $( \pm 0,93)$ & $( \pm 0,11)$ & $( \pm 0,11)$ & $( \pm 6,94)$ & $( \pm 3,03)$ \\
\hline \multirow{2}{*}{2} & $12,58 a$ & $0,34 a$ & $0,95 a$ & $13,07 \mathrm{ab}$ & $1,55 a$ & $0,99 a$ & $417,33 a$ & $33,76 a b$ \\
\hline & $( \pm 1,14)$ & $( \pm 0,03)$ & $( \pm 0,14)$ & $( \pm 0,75)$ & $( \pm 0,14)$ & $( \pm 0,16)$ & $( \pm 4,67)$ & $( \pm 3,29)$ \\
\hline \multirow{2}{*}{3} & $14,2 \mathrm{a}$ & $0,36 a$ & $0,91 a$ & $14,22 a b$ & $1,63 a$ & $1,12 a$ & $398,33 a$ & $28,07 b$ \\
\hline & $( \pm 0,67)$ & $( \pm 0,03)$ & $( \pm 0,14)$ & $( \pm 0,75)$ & $( \pm 0,17)$ & $( \pm 0,09)$ & $( \pm 19,70)$ & $( \pm 1,07)$ \\
\hline \multirow{2}{*}{4} & $13,06 a$ & $0,34 a$ & $0,77 a$ & $17,37 a$ & $1,77 a$ & $1,01 a$ & $420,00 a$ & $32,22 a b$ \\
\hline & $( \pm 0,48)$ & $( \pm 0,01)$ & $( \pm 0,01)$ & $( \pm 1,07)$ & $( \pm 0,07)$ & $( \pm 0,06)$ & $( \pm 7,23)$ & $( \pm 0,94)$ \\
\hline $\mathrm{CV}(\%)^{(2)}$ & 11,56 & 13,19 & 26,29 & 12,24 & 13,4 & 19,64 & 4,87 & 12,23 \\
\hline
\end{tabular}


O macronutriente que apresentou o maior teor foi Ca, pelo fato de serum componente estrutural das células do tecido vegetal e, deste modo, é um dos últimos nutrientes a ser liberado para o solo via decomposição da serapilheira. Segundo Clevelario Jr. (1996), o enriquecimento em Ca da serapilheira pode ser decorrente de uma liberação mais lenta deste elemento pelo material recém-caído, da translocação de outros elementos antes da abscisão das folhas, da redução da massa das folhas antes da abscisão e ou consequência da retenção de Ca contido na transprecipitação (chuva que atravessa o dossel) pela serapilheira. Sendo ainda seu elevado teor associado ao grande aporte em diversas florestas tropicais, com valores mais elevados ao final da estação seca, e redução nos meses mais chuvosos (Vitousek, 1984; Vital, et al., 2004; Caldeira, et al 2008).

A ciclagem biogeoquímica, de modo geral, é a via pela qual os nutrientes de baixa mobilidade na planta, como Ca, são ciclados, uma vez que para esses nutrientes a ciclagem bioquímica torna-se pouco expressiva, contrariamente ao que ocorre para nutrientes de alta mobilidade na planta (Caldeira et al., 2008).

Apesar de não significativos, o maior teor de $\mathrm{N}$ no tratamento T3 $\left(14,20 \mathrm{~g} \mathrm{~kg}^{-1}\right)$ em relação aos demais tratamentos pode estar relacionado à presença de Sesbania grandiflora. Espécies de leguminosas são amplamente utilizadas para melhorar a qualidade de solos em projetos de recuperação, principalmente por contribuírem com grande aporte de serapilheira, e com a fixação biológica de $\mathrm{N}$.

A influência positiva desta espécie no T3 pode ser confirmada pela diferença significativa existente para a relação $\mathrm{C} / \mathrm{N}$, onde os menores valores foram observados para este tratamento.

Como os valores da relação $\mathrm{C} / \mathrm{N}$ do material acumulado nos tratamentos T1, T2 e T4 são maiores que 30, a imobilização é superior a mineralização (Stevenson, 1986), fazendo com que a disponibilidade de $\mathrm{N}$ seja diminuída. Ou seja, a serapilheira causa imobilização líquida de N. A disponibilidade de $N$ não é alterada para o tratamento T3, onde há Sesbania grandiflora, sendo a relação $\mathrm{C} / \mathrm{N}$ entre 20 e 30 (Stevenson, 1986).

Juntamente com 0 processo de mineralização ocorre a imobilização dos nutrientes a fim de atender a demanda nutricional dos organismos decompositores que regulam a disponibilidade liquidas de nutrientes, esse balanço depende da qualidade do resíduo que está estreitamente ligado ao teor de $\mathrm{N}$ dos resíduos (Godinho et al. 2014).

Os teores de micronutrientes na serapilheira acumulada apresentaram a seguinte ordem decrescente: $\mathrm{Fe}>\mathrm{Mn}>\mathrm{Zn}>\mathrm{B}>$ Cu (Tabela 4).

Tabela 4. Teores médios dos micronutrientes da serapilheira acumulada nos diferentes métodos de restauração florestal de Mata Atlântica de tabuleiros, Linhares - ES.

\begin{tabular}{|c|c|c|c|c|c|}
\hline \multirow{2}{*}{ Tratamento } & $B$ & $\mathrm{Cu}$ & $\mathrm{Fe}$ & $M n$ & $\mathrm{Zn}$ \\
\hline & \multicolumn{5}{|c|}{$\mathrm{mg} \mathrm{kg}^{-1}$} \\
\hline 1 & $\begin{array}{l}32,85 a^{*} \\
( \pm 3,25)^{(\prime)}\end{array}$ & $\begin{array}{l}14,73 a \\
( \pm 0,88)\end{array}$ & $\begin{array}{c}880,37 a \\
( \pm 182,20)\end{array}$ & $\begin{array}{c}175,59 a \\
( \pm 8,66)\end{array}$ & $\begin{array}{l}43,67 \mathrm{a} \\
( \pm 2,41)\end{array}$ \\
\hline 2 & $\begin{array}{l}27,72 a \\
( \pm 3,34)\end{array}$ & $\begin{array}{l}13,69 a \\
( \pm 0,83)\end{array}$ & $\begin{array}{c}949,12 a \\
( \pm 175,23)\end{array}$ & $\begin{array}{l}128,61 \mathrm{a} \\
( \pm 32,74)\end{array}$ & $\begin{array}{l}34,25 b \\
( \pm 1,40)\end{array}$ \\
\hline 3 & $\begin{array}{l}29,16 a \\
( \pm 2,47)\end{array}$ & $\begin{array}{l}14,21 a \\
( \pm 1,22)\end{array}$ & $\begin{array}{l}1510,15 a \\
( \pm 304,27)\end{array}$ & $\begin{array}{c}151,28 a \\
( \pm 9,44)\end{array}$ & $\begin{array}{c}36,40 \mathrm{ab} \\
( \pm 2,07)\end{array}$ \\
\hline 4 & $\begin{array}{l}27,45 a \\
( \pm 1,31)\end{array}$ & $\begin{array}{l}11,98 \mathrm{a} \\
( \pm 1,17)\end{array}$ & $\begin{array}{l}1218,69 a \\
( \pm 267,63)\end{array}$ & $\begin{array}{l}125,64 a \\
( \pm 10,71)\end{array}$ & $\begin{array}{l}34,08 \mathrm{~b} \\
( \pm 0,11)\end{array}$ \\
\hline $\mathrm{CV}(\%)^{(2)}$ & 15,54 & 12,63 & 41,7 & 20,56 & 8,72 \\
\hline
\end{tabular}

Nesse estudo somente o Zn apresentou diferença significativa com maior teor médio para o tratamento $\mathrm{Tl}$ e menor para os tratamentos T2 e T4 (Tabela 4). Caldeira et al. (2007), relatam que os maiores teores e conteúdos, principalmente de $\mathrm{Fe}$ e $\mathrm{Zn}$ na serapilheira acumulada, podem ser justificados pela sua mobilidade e, consequentemente, pelos seus maiores teores nas folhas de algumas espécies, bem como maiores teores médios nas folhas da 
floresta em relação à madeira, casca e galhos. Outra justificativa pode ser a contaminação com o solo, ou seja, amostras de serapilheira com solo. Ainda segundo Vieira e Schumacher (2010), altos teores de Fe e Mn, podem significar teores elevados desses nutrientes no solo.
Conteúdo de nutrientes

Os macronutrientes $\mathrm{Ca}$ e $\mathrm{P}$, respectivamente, apresentaram maior e o menor conteúdo para os tratamentos estudados (Tabela 5), sendo a ordem decrescente dos conteúdos foi: $\mathrm{Ca}>\mathrm{N}>\mathrm{K}>\mathrm{Mg}>\mathrm{S}>\mathrm{P}$.

Tabela 5. Conteúdo de macronutrientes e carbono orgânico na serapilheira acumulada nos diferentes métodos de restauração florestal de Mata Atlântica de tabuleiros, Linhares - ES.

\begin{tabular}{|c|c|c|c|c|c|c|c|}
\hline \multirow{2}{*}{ Tratamentos } & $\mathrm{N}$ & $\mathrm{P}$ & $K$ & $\mathrm{Ca}$ & $\mathrm{Mg}$ & $S$ & $\mathrm{CO}$ \\
\hline & \multicolumn{7}{|c|}{$\left(\mathrm{kg} \mathrm{ha}^{-1}\right)$} \\
\hline \multirow{2}{*}{1} & $108,98 a^{*}$ & $2,94 a$ & $11,65 a$ & $127,52 a$ & $19,92 a$ & $9,71 a$ & $4353,05 a$ \\
\hline & $( \pm 12,76)^{(1)}$ & $( \pm 0,12)$ & $( \pm 1,52)$ & $( \pm 21,18)$ & $( \pm 2,87)$ & $( \pm 1,18)$ & $( \pm 309,08)$ \\
\hline \multirow{2}{*}{2} & $101,61 a$ & $2,72 a$ & $7,46 a b$ & $104,00 a$ & $12,34 a$ & $8,11 a$ & $3303,63 a$ \\
\hline & $( \pm 23,09)$ & $( \pm 0,52)$ & $( \pm 1,51)$ & $( \pm 19,86)$ & $( \pm 2,45)$ & $( \pm 2,22)$ & $( \pm 602,37)$ \\
\hline \multirow[b]{2}{*}{3} & $126,04 a$ & $3,16 a$ & $8,10 a b$ & $132,69 a$ & $15,14 a$ & $9,78 \mathrm{a}$ & $3526,07 a$ \\
\hline & $( \pm 29,30)$ & $( \pm 0,63)$ & $( \pm 2,03)$ & $( \pm 42,30)$ & $( \pm 4,51)$ & $( \pm 2,11)$ & $( \pm 775,50)$ \\
\hline \multirow{2}{*}{4} & $92,43 a$ & $2,47 a$ & $5,49 b$ & $122,15 a$ & $12,66 a$ & $7,12 a$ & $2992,63 a$ \\
\hline & $( \pm 8,04)$ & $( \pm 0,35)$ & $( \pm 0,65)$ & $( \pm 7,90)$ & $( \pm 1,65)$ & $( \pm 0,52)$ & $( \pm 336,17)$ \\
\hline $\mathrm{CV}(\%)^{(2)}$ & 28,5 & 32,61 & 27,25 & 23,35 & 40,06 & 37,49 & 30,53 \\
\hline
\end{tabular}

O $\mathrm{K}$ foi o que apresentou diferença estatística, apresentando média superior para o tratamento $\mathrm{Tl}$ é menor para o tratamento T4 (Tabela 5). Esse fato pode ser explicado pela predominância de gramíneas no tratamento $\mathrm{Tl}$ que, de acordo com Troeh \& Thompson (2007), absorvem grandes quantidades de $\mathrm{K}$.

Dentre os macronutrientes, O Ca apresentou o maior conteúdo na serapilheira acumulada, fato este que pode estar relacionado com a sua pouca mobilidade nos tecidos vegetais. A baixa mobilidade deste macronutriente dentro dos tecidos vegetais é citada por Attiwill (1979) e Nilsson et al. (1995) e como um fator que determina que a maior quantidade de ciclagem deste nutriente na natureza seja feita pela queda e decomposição dos tecidos vegetais senescentes.

Segundo Caldeira et al. (2008), a serapilheira acumulada é a principal via de transferência de $\mathrm{N}, \mathrm{K}$ e Ca para o solo. Quantidades significativas de nutrientes $\mathrm{e}$ carbono orgânico podem retornar ao solo através da queda de componentes senescentes da parte aérea das plantas e sua posterior decomposição. Os valores do conteúdo de macronutrientes observados nesse estudo são superiores aqueles observados pelo referido autor em uma Floresta Ombrófila Densa Submontana para estádio inicial e intermediário.

Não foi encontrada diferença estatística para o conteúdo de micronutrientes na serapilheira acumulada entre os tratamentos estudados (Tabela 6). A floresta em estudo apresentou para o conteúdo médio de micronutrientes, a seguinte ordem decrescente: $\mathrm{Fe}>\mathrm{Mn}>\mathrm{B}>\mathrm{Zn}>\mathrm{Cu}$.

Os maiores teores e conteúdos, principalmente de Fe na serapilheira acumulada podem ser justificados pela sua mobilidade. Segundo Dechen \& Nachtigall (2006) e Malavolta (2006), em relação ao metabolismo do $\mathrm{Fe}$ na planta, deve-se levar em conta que este apresenta baixa mobilidade nos tecidos vegetais. Essa mobilidade é afetada, negativamente, por vários fatores, como elevado conteúdo de $P$, deficiência de $K$, quantidade elevada de Mn e baixa intensidade luminosa (Dechen \& Nachtigall, 2006). A outra justificativa pode ser em função dos maiores teores nas folhas velhas de algumas espécies, bem como teores médios maiores nas folhas da floresta em relação a madeira, casca e galhos (Caldeira et al., 2008). A contaminação com o solo, ou seja, amostra de serapilheira com solo pode ser considerada outra justificativa. Os conteúdos de argila e matéria orgânica no solo influenciam também na disponibilidade do Fe (Dechen \& Nachtigall, 
2006).

O segundo micronutriente com maior conteúdo na serapilheira acumulada é $\mathrm{Mn}$ (Tabela 6). Esse fato pode ser também em função da contaminação com o solo, ou seja, amostra de serapilheira com solo, pois $\mathrm{Mn}$ no solo é proveniente de óxidos, carbonatos, silicatos e sulfetos (Dechen \& Nachtigall, 2006). Cabe ressaltar que maiores teores e conteúdos de $\mathrm{Mn}$ na serapilheira acumulada podem ser justificados pelos seus maiores teores nas folhas de algumas espécies (Caldeira et al., 2008).
O B é o terceiro micronutriente em conteúdo na serapilheira acumulada (Tabela 6), sendo que esse fato pode estar relacionado com a sua pouca mobilidade nos tecidos vegetais. Deste modo, quanto mais velha a folha maior o teor de B (Jones Júnior, 1970). A mobilidade intermediária do $\mathrm{B}$ dentro dos tecidos vegetais (mobilidade intermediária no floema) (Marschner, 1997) pode ser um dos fatores que determinam que a maior intensidade de ciclagem deste nutriente na natureza seja produzida pela queda e decomposição dos tecidos senescentes.

Tabela 6. Conteúdo médio de micronutrientes na serapilheira acumulada nos diferentes métodos de restauração florestal de Mata Atlântica de tabuleiros, Linhares - ES.

\begin{tabular}{|c|c|c|c|c|c|}
\hline \multirow{2}{*}{ Tratamento } & $B$ & $\mathrm{Cu}$ & $\mathrm{Fe}$ & $M n$ & $\mathrm{Zn}$ \\
\hline & \multicolumn{5}{|c|}{$\left(\mathrm{kg} \mathrm{ha}^{-1}\right)$} \\
\hline \multirow{2}{*}{1} & $0,33 a^{*}$ & $0,15 a$ & $9,32 a$ & $1,81 a$ & $0,45 a$ \\
\hline & $( \pm 0,01)^{(1)}$ & $( \pm 0,00)$ & $( \pm 2,52)$ & $( \pm 0,17)$ & $( \pm 0,03)$ \\
\hline \multirow{2}{*}{2} & $0,21 a$ & $0,11 a$ & $7,98 a$ & $1,11 a$ & $0,27 a$ \\
\hline & $( \pm 0,02$ & $( \pm 0,01)$ & $( \pm 2,64)$ & $( \pm 0,43)$ & $( \pm 0,06)$ \\
\hline \multirow{2}{*}{3} & $0,27 a$ & $0,12 a$ & $14,06 a$ & $1,41 a$ & $0,33 a$ \\
\hline & $\pm 0,08$ & $( \pm 0,02)$ & $( \pm 5,67)$ & $( \pm 0,46)$ & $( \pm 0,09)$ \\
\hline \multirow{2}{*}{4} & 0,19 & $0,09 a$ & $8,34 a$ & $0,88 a$ & $0,24 a$ \\
\hline & $( \pm 0,02$ & $( \pm 0,02)$ & $( \pm 1,18)$ & $( \pm 0,07)$ & $( \pm 0,03)$ \\
\hline $\mathrm{CV}(\%)^{(2)}$ & 25,97 & 27,99 & 65,14 & 44,38 & 31,21 \\
\hline
\end{tabular}

\section{Conclusões}

Os atributos químicos do solo, bem como, o conteúdo de nutrientes da serapilheira não sofreu alterações pela utilização de diferentes metodologias de restauração, ou mesmo pela diversidade de espécies presentes.

A implantação de plantios para recuperação de áreas degradadas, com a presença de espécies leguminosas, em especial a Sesbania grandiffora, propicia um material senescente de melhor qualidade e baixa relação $\mathrm{C} / \mathrm{N}$.

\section{Agradecimentos}

A Reserva Natural Vale, Linhares - ES, pela disponibilidade da área do estudo e pela concessão da bolsa de mestrado.

\section{Referências}

Attiwill, P.M. Nutrient scling in eucalyptus and pinus forests in Southeastern Australia. In: Anais Of Symposium on Impact of Intensive Harvesting on Forest Nutrient Cycling, New York, 1979.
Caldeira, M.V.W., Marques, R., Soares, R.V., Balbinot, R. 2007. Quantificação de serapilheira e de nutrientes - Floresta Ombrófila Mista Montana - Paraná. Revista Acadêmica 5: 101-116.

Caldeira, M.V.W., Vitorino, M.D., Schaadt, S.S., Moraes, E., Balbinot, R. 2008. Quantificação de serapilheira e de nutrientes em uma Floresta Ombrófila Densa. Semina: Ciências Agrárias 29: 53-68.

Caló, L. O. 2014. Atributos químicos e biológicos do solo em áreas de restauração florestal. $110 f$. (Dissertação de Mestrado) - Universidade Federal do Espírito Santo, Jeronimo Monteiro, Brasil.

Chada, S. S.; Campello, E.F.C.; Faria, S.M. 2004. Sucessão vegetal em uma encosta reflorestada com leguminosas arbóreas em Angra dos Reis, Revista Árvore, Viçosa-MG, 28(6): 801-809.

Clevelario Junior, J. 1996. Distribuição de carbono e elementos minerais em um ecossistema de floresta tropical úmido baixo montano. Viçosa, MG. 150f. (Tese de Doutorado) - Universidade Federal de Viçosa, Viçosa, Brasil.

Cuevas, E.; Medina, E. 1986. Nutrient dynamics within Amazonian Forest ecosystems. In: .Nutrient flux in the fine litterfall and efficiency of 
nutrienteutilization. Oecologia 68: 466-472.

Dechen, A.R., Nachtigall, G.R. 2006. Micronutrientes. In. Fernandes, M.S. (Ed). Nutrição mineral de plantas. Viçosa: Sociedades Brasileiras de Ciência do Solo, p. 327-374.

EMBRAPA. Centro Nacional de Pesquisa de Solos (Rio de Janeiro). Sistema Brasileiro de Classificação de solos. 2.ed. Rio de Janeiro 2006. $306 \mathrm{p}$.

Fernandes, M.E.B., Nascimento, A.A.M., Carvalho, M.L. 2007. Estimativa da produção anual de serapilheira dos bosques de mangue no Furo Grande, Bragança-Pará. Revista Árvore 31: 949958.

Figueiredo Filho, A., Moraes, G.F., Schaaf, L.B., Figueiredo, D.J. 2003. Avaliação estacional da deposição de serapilheira em uma floresta Ombrófila Mista localizada no sul do estado do Paraná. Ciência Florestal 13: 11-18.

Franco, A.A., Faria, S.M. 1997. The contribution of $\mathrm{N}_{2}$-fixing tree legumes to land reclamation and sustainability in the tropics. Soil Biochemistry 29: 897-983.

Gama-Rodrigues, A.C., Barros, N.F., Mendonça, E.S. 1999. Alterações edáficas sob plantios puros e mistos de espécies florestais nativas do Sudeste da Bahia, Brasil. Revista Brasileira de Ciência do Solo 23: 581-592.

Godinho, T. O., Caldeira, M. V. W., Rocha, J. H. T., Caliman, J. P., \& Trazzi, P. A. 2014. Quantificação de biomassa e nutrientes na serapilheira acumulada em trecho de Floresta Estacional Semidecidual Submontana, ES. Revista Cerne, 20(1), 11-20.

IBGE. 2012. Manual técnico da vegetação brasileira. Instituto Brasileiro de Geografia e Estatística. Rio de Janeiro, 275 p.

Jesus, R.M., Rolim, S.G. 2005. Fitossociologia da Mata Atlântica de Tabuleiro. Viçosa: SIF, Boletim técnico SIF 19: 1-149.

Jones Júnior, J. B. 1970. Distribution if 15 elements in corn leaves. Communications in Soil Science and Plant Analysis, New York 1 : 27-34.

Köppen, W. 1948. Climatologia: con un estudio de los climas de la tierra. Fondo de Cultura Econômica. México. 479 p.

Magnago, L. F. S., Simonelli, M., Martins, S. V., Matos, F. A. R., \& Demuner, V. G. 2011 . Variações estruturais e características edáficas em diferentes estádios sucessionais de Floresta Ciliar de Tabuleiro, ES. Revista Árvore, 35(3), 445-456.

Malavolta, E. 2006. Manual de nutrição de plantas. São Paulo: Agronômica Ceres, 638 p.

Marschner, H. 1997. Mineral nutrition of higher pants. 2 ed. San Diego: Academic, 889 p.

Moreau, J. S. 2014. Estrutura e interação entre vegetação e ambiente de uma Floresta Ombrófila Densa das Terras Baixas, Espírito Santo. 96f. (Dissertação de Mestrado) - Universidade Federal do Espírito Santo, Jeronimo Monteiro, Brasil.

Moreira, F. L. 2014. Ciclagem de nutrientes em área de restauração de Floresta Ombrófila Densa das Terras Baixas. 105f. (Dissertação de Mestrado) - Universidade Federal do Espírito Santo, Jeronimo Monteiro, Brasil.

Nilsson, L.O., Huttl R.F., Johansson, U.T., Jochheim, H. 1995. Nutrient uptake and cycling in forest ecosystems - present status and future research directions. Plant and Soil, The Hague 168/169: 5-13.

O'Connell, A.M., Sankaran, K.V. 1997. Organic matter accretion, decomposition and mineralisation. In: Nambiar, E.K.S., Brown, A.G. (Ed.) Management of soil, nutrients and water in tropical plantations forests. ACIAR, Canberra, Australia, p. 443-480.

Poggiani, F., Schumacher, M.V. 2000. Ciclagem de nutrientes em florestas nativas. In: Gonçalves, J.L.M., Benedetti, V. Nutrição e fertilização florestal. IPEF, p. 287 - 308.

Rodrigues, R.R., Gandolfi, S., Nave, A. G. 2002. Adequação ambiental de propriedades rurais e recuperação de áreas degradadas. ESALQ, Piracicaba, Brasil, 63.

Stevenson, F. J. (ed.). 1986. Nitrogen in agricultural soils. Madison, American Society of Agronomy. 940p.

Tedesco, M.J., Gianello, C., Bissani, C.A., Bohnen, H., Volkweiss, S.J. 1995. Análises de solos, plantas e outros materiais. 2 ed., UFRGS Porto Alegre, Brasil, 174p. (Boletim Técnico, 5).

Troeh, F.R., Thompson, L.M. 2007. Solos e fertilidade do solo. 6 ed., Ed. Andrei, São Paulo, Brasil, 717 p.

Viera, M., Schumacher, M.V. 2010. Teores e aporte de nutrientes na serapilheira de Pinus taeda L., e sua relação com a temperatura do ar e pluviosidade. Revista Árvore 34: 85-94.

Vital, A.R.T., Guerrini, I.A., Franken, W.K., Fonseca, R.C.B. 2004. Produção de serapilheira e ciclagem de nutrientes de uma Floresta Estacional Semidecidual em zona ripária. Revista Árvore 28: 793-800.

Vitousek, P.M. 1984. Litterfall, nutrient cycling, and 
nutrient limitation in tropical forests. Ecology 65: 285-298.

Wedderburn, M.E.; Carter, J. 1999. Litter decomposition by four functional tree types for use in silvopastoral systems. Soil Biology and Biochemistry 31: 455-461. 